\title{
How intent to interact can affect action scaling of distance: reply to Wilson
}

\author{
Tamer M. Soliman and Arthur M. Glenberg* \\ Department of Psychology, Arizona State University, Tempe, AZ, USA \\ ${ }^{*}$ Correspondence: glenberg@asu.edu
}

Edited and reviewed by:

Guy Dove, University of Louisville, USA

Keywords: culture, self-construal, embodied cognition, distance perception, motor effort

\section{A commentary on}

Action scaling of distance perception is task specific and does not predict "the embodiment of culture": a comment on Soliman, Gibson and Glenberg (2013) by Wilson, A. D. (2014). Front. Psychol. 5:302. doi: 10.3389/fpsyg.2014.00302

Soliman et al. (2013) set out to demonstrate how the bodily level of analysis can unify explanations in psychology. Our argument was that common sensorimotor mechanisms underlie many of the behavioral phenomena that are currently segregated as cognitive, social, or cultural. Toward that end, we re-characterized a cultural construct-self-construal along the dimension of independence and interdependence (Markus and Kitayama, 1991)_ as reflecting degree of interaction with ethnically diverse others.

According to our cultural motor-effort hypothesis, the interdependenceindependence continuum is in part determined by tuning sensorimotor behavior through interactions. Interdependents tune vocal, gestural, expressive facial patterns, as well as interactions in greeting, eating, walking, dancing, praying (and so on) with members of their in-groups. In contrast, independents tune their interactions with a more ethnically-diverse set of people. Consequently, interdependents, more so than independents, would anticipate greater motor effort when interacting with out-groups (vs. in-groups) because of poor tuning. Furthermore, reasoning from Proffitt and Linkenauger (2013) as well as Schnall et al. (2008), anticipated motor effort should lead to increased distance judgments. Thus we predicted, and found, that interdependents judge distance to in-group members as shorter than do independents.

Wilson (2014) questioned our application of Proffitt and Linkenauger and Schnall et al. As he notes, Proffitt's data (although not data from Schnall et al.) suggest that effects of anticipated motor effort are restricted to particular motor systems. Hence, Wilson reasoned, the anticipated effort in interacting should not affect scaling of distance when planning to walk. Here we address Wilson's reasoning by (a) pointing to several research projects that suggest leakage across motor systems rather than modularity, and (b) suggesting why previous data, importantly, Witt et al. $(2004,2010)$ did not observe this leakage.

As one example of leakage, consider data reported by Gentilucci et al. (2001). When reaching for a block, the larger the block, the wider people unintentionally open their mouths. In addition, the larger the block, the louder they pronounce syllables printed on the block.

Now consider in more detail retroactive motor contagion (RMC): the ubiquitous finding that if two action patterns are conjoined, planning of the second action influences planning of the first action. Demonstrations of RMC can be found in Adam et al. (2000), Khan et al. (2007, 2010), and Lajoie and Franks (1997).

The "end-comfort effect" can also be seen as a type of RMC. For example, the kinematics of the transport-to-grasp movement toward a bottle systematically vary depending on whether the bottle is later to be displaced to a different spot, is used to pour water into a glass, or is to be thrown away (Ansuini et al., 2008). Importantly, RMC can cross anatomical and neuro-representational boundaries within the motor system and shows coordination across different effectors. van der Wel and Rosenbaum (2007), for example, asked participants to locomote to a table, grasp a bottle, and then move it to another spot that was either close to or far from its initial location. The initial motor pattern (i.e., locomotion) was found to be influenced by the distal motor pattern (i.e., object transport). Namely, a participant's final step was on the side opposite to the direction of the forthcoming transport movement when that transport required one more step after grasping (see also Cockell et al., 1995 and Studenka et al., 2012).

Thus, modularity of the motor system at the anatomical and brainrepresentational level does not always hold at the functional level. Instead, conjoining two action patterns induces an informational flow across effectors and planned goals. Importantly, this influence holds whether one or different motor systems are involved in the sequence, and whether the goals planned are homologous (e.g., tapping followed by tapping) or different (i.e., locomoting then grasping). In short, these findings support our assumption that anticipated effort of interaction can affect anticipated effort to walk, and thereby affect distance judgments.

With the above as a backdrop, why then do Proffitt's data (e.g., Witt et al., 2004, 2010) seem to suggest modularity? One possibility is based on a subtle difference between the design of our experiments and the Witt et al. experiments. In Witt's experiments, the manipulation phase targets one motor system and then tests the effect of the manipulation on perceived distance as the participant intends to 
perform another task. For example, adapting Proffitt and Linkenauger's (2013) terminology, participants are adapted while temporarily turned into throwing phenotypes, and then tested while in the walking phenotype. Typically, it was found that the visio-motor scale developed while in one phenotype did not transfer to the other: the thrower-turned-walker participants do not show effects of the earlier throwing manipulation on their distance judgments while walkers (Witt et al., 2004), and vice versa (Witt et al., 2010).

In our experiments, however, no behavioral phenotype was turned on, manipulated, switched off, replaced by another, and then examined. Instead, our participants were walker-then-interactor phenotypes throughout. That is, the phenotype we manipulated (the interactor phenotype) was (a) always turned on and (b) always conjoined with the walker phenotype. Thus, by virtue of being conjoined with the interaction system during simulation, the locomotion system was "contaminated" by the constantly-running interaction system. This conjoining led to the effort parameter values instantiated in the interaction system to diffuse into the parameters in the locomotion system. We captured the state of the latter parameters through visual-distance estimates, and we hypothesized that they function, by proxy, as indicators of the amount of effort experienced by the interaction system.

We believe that these subtle design differences render our original results and theoretical arguments immune to Wilson's critiques. Perceived motor effort to interact with in-groups and outgroups can still be a conceptually valid re-characterizations of the cultural construct of interdependence-independence. And, importantly, when viewed in light of the RMC effects, our results can be categorized as belonging to the same class of phenomena explained by Proffitt's theoretical framework. We thank Wilson for providing the opportunity for us to develop this account in greater detail, and we look forward to tests of the proposal.

\section{ACKNOWLEDGMENTS}

Tamer Soliman was support by a Ford Foundation Fellowship and Seed Funding from the College of Liberal Arts and Sciences, and Arthur Glenberg was partially supported by NSF grants 1020367 and1324807. Any opinions, findings, and conclusions or recommendations expressed in this material are those of the authors and do not necessarily reflect the views of the funding agencies.

\section{REFERENCES}

Adam, J. J., Nieuwenstein, J. H., Huys, R., Paas, F. G., Kingma, H., Willems, P., et al. (2000). Control of rapid aimed hand movements: the one- target advantage. J. Exp. Psychol. Hum. Percept. Perform. 26, 295-312. doi: 10.1037/0096-1523.26.1.295

Ansuini, C., Giosa, L., Turella, L., Altoe, G., and Castiello, U. (2008). An object for an action, the same object for other actions: effects on hand shaping. Exp. Brain Res. 185, 111-119. doi: 10.1007/s00221-007-1136-4

Cockell, D. L., Carnahan, H., and McFadyen, B. J. (1995) A preliminary analysis of the coordination of reaching, grasping, and walking. Percept. Mot. Skills 81, 515-519. doi: 10.2466/pms.1995. 81.2.515

Gentilucci, M., Benuzzi, F., Gangitano, M., and Grimaldi, S. (2001). Grasp with hand and mouth: a kinematic study on healthy subjects. $J$. Neurophysiol. 86, 1685-1699.

Khan, M., Mottram, T., Adam, J., and Buckolz, E. (2010). Sequential movement with two limbs and the one target advantage. J. Mot. Behav. 42, 325-332. doi: 10.1080/00222895.2010. 510544

Khan, M. A., Mourton, S., Buckolz, E., and Franks, I. M. (2007). The influence of advance information on the response complexity effect in manual aiming movements. Acta. Psychol. (Amst), 127, 154-162. doi: 10.1016/j.actpsy.2007. 04.001

Lajoie, J. M., and Franks, I. M. (1997). Response programming as a function of accuracy and complexity: evidence lfom latency and kinematic measures. Hum. Mov. Sci. 16, 485-505.

Markus, H., and Kitayama, S. (1991). Culture and the self: implications for cognition, emotion, and motivation. Psychol. Rev. 98, 224-253. doi: 10.1037/0033- 295X.98.2.224

Proffitt, D. R., and Linkenauger, S. A. (2013). "Perception viewed as a phenotypic expression," in Action Science: Foundations of An Emerging Discipline, eds W. Prinz, M. Beisert, and A. Herwig (Cambridge, MA: MIT Press), 171-197.

Schnall, S., Harber, K., Stefanucci, J., and Proffitt, D. (2008). Social support and the perception of geographical slant. J. Exp. Soc. Psychol. 44, 1246-1255. doi: 10.1016/j.jesp.2008.04.011

Soliman, T., Gibson, A., and Glenberg, A. M. (2013) Sensory motor mechanisms unify psychology: the embodiment of culture. Front. Psychol. 4:885. doi: 10.3389/fpsyg.2013.00885

Studenka, B. E., Seegelke, C., Schutz, C., and Schack, T. (2012) Posture based motor planning in a sequential grasping task. J. Appl. Res. Mem. Cogn. 1, 89-95. doi: 10.1016/j.jarmac.2012.02.003

Wilson, A. D. (2014). Action scaling of distance perception is task specific and does not predict "the embodiment of culture": a comment on Soliman, Gibson and Glenberg (2013). Front. Psychol. 5:302. doi: 10.3389/fpsyg.2014.00302

Witt, J. K., Proffitt, D. R., and Epstein, W. (2004). Perceiving distance: a role of effort and intent. Perception 33, 570-590. doi: 10.1068/p5090

Witt, J. K., Proffitt, D. R., and Epstein, W. (2010). When and how are spatial perceptions scaled? J. Exp. Psychol. Hum. Percept. Perform. 36, 1153-1160. doi: 10.1037/a0019947

van der Wel, R. P., and Rosenbaum, D. A. (2007). Coordination of locomotion and prehension. Exp. Brain Res. 176, 281-287. doi: 10.1007/s00221-0060618-0

Conflict of Interest Statement: The authors declare that the research was conducted in the absence of any commercial or financial relationships that could be construed as a potential conflict of interest.

Received: 08 April 2014; accepted: 10 May 2014; published online: 05 June 2014.

Citation: Soliman TM and Glenberg AM (2014) How intent to interact can affect action scaling of distance: reply to Wilson. Front. Psychol. 5:513. doi: 10.3389/ fpsyg.2014.00513

This article was submitted to Cognitive Science, a section of the journal Frontiers in Psychology.

Copyright (c) 2014 Soliman and Glenberg. This is an open-access article distributed under the terms of the Creative Commons Attribution License (CC BY). The use, distribution or reproduction in other forums is permitted, provided the original author(s) or licensor are credited and that the original publication in this journal is cited, in accordance with accepted academic practice. No use, distribution or reproduction is permitted which does not comply with these terms. 